\title{
ANGINA PECTORIS WITH ASSOCIATED LEFT PAROXYSMAL PTOSIS
}

\author{
BY \\ H. G. MILLER AND RAYMOND DALEY \\ From the Medical Division of Princess Mary's Royal Air Force Hospital, Halton \\ Received October 12, 1945
}

The present case is reported because it presents what appears to be a unique combination of clinical phenomena.

An officer, 48 years of age, was well until August 1943. At this time, and at the end of a strenuous ten-day battle course, he was climbing a high wall when he had a sudden attack of severe pain in the chest accompanied by breathlessness. The pain did not radiate, but was generalized over the sternum and all the ribs on the left side anteriorly. He felt as though his chest was gripped in a vice and was very badly shaken, and stayed in bed for two days. A few days later he was "dressing down" one of his subordinates and at the same time vigorously demonstrating how a job should be done, when he suddenly felt an intense pain in the xiphisternum accompanied by severe dyspnoea. He had to stop talking and sit down at once and the pain slowly disappeared during the course of the next ninety minutes. At 3.30 the next morning he was awakened by a severe pain in the left arm, beginning in the elbow and spreading down the dorsum of the arm to all the knuckles; it was particularly acute in the thenar muscles. At the same time he became aware, though less insistently, of a mid-sternal pain, but on this occasion the arm pain was the more severe and was described as being like electric shocks passing down the forearm in rapid succession, rendering the hand practically powerless. He slept no more that morning but by breakfast time the pain was gone except for a dull ache in forearm and sternum. From this time until mid-December he had constant aching discomfort in the chest and left arm which turned to severe pain on moderate exertion. The continual pain in the arm was not well localized except that it was all below the elbow. Exertion, however, produced well-defined pain and tingling in the ulnar forearm and fingers, and if the exertion was continued the pain spread to the radial side of the hand and to the thenar muscles.

One day in October he was cycling uphill and already feeling rather breathless when he suddenly became aware that his left eye was running and that tears were streaming down his face on this side. He attributed this to the cold wind, and when he dismounted his bicycle, five minutes later, there was a burning pain on the left side of his face affecting chiefly the temple, eye, cheek, and angle of the lower jaw. At the same time he noticed that his left face felt warm and sticky. He lectured for nearly an hour in this condition and was then suddenly seized by a severe pain, which was mid-sternal and involved the whole of the left forearm and hand simultaneously. This was as severe as any attack he had ever suffered, but it disappeared after half an hour's rest and within an hour his eye had stopped running.

From this time on all the severe attacks began in the same way, with watering of the left eye followed by pain in the left face which was observed to be warm, congested, and moist to the touch. He noticed that if he sat down at the onset of lachrymation the subsequent chest pain was either much less severe or, on some occasions, did not come on at all; and that the onset of attacks with running of the left eye bore a constant relation to exertion, occurring always during the same hundred yards of his uphill journey from his place of work. If he exerted himself severely indoors the eyes watered in the same way, though, of course, he was 
more commonly outside when this happened. When the exertion was violent and sudden, as in cycling up a steep hill against the wind, and particularly after lunch, the chest and arm pain sometimes occurred immediately, without lachrymation or facial pain, but on those occasions the latter symptoms began a few moments later and usually together.

He continued thus until mid-December. One afternoon just as he was recovering from an attack of post-prandial exertional pain he had his photograph taken. When the picture came back from the printers, he noticed that his left eye was practically closed. This was the first occasion on which ptosis was noted, and after this time he observed a gradual drooping of the left lid simultaneously with the onset of the other ocular symptoms in every attack. The ptosis was such that in a severe attack he could not see except by raising the lid manually.

In mid-December the constant chest and arm pain cleared, but he still had frequent exertional attacks of the same type and severity as before. They began with weeping and pain in the periphery of the left face and with flickering in the upper and lower lids, after which the upper lid began to droop. Usually about half an hour later pain began simultaneously in the chest and arm. This period was inversely proportional to the severity of the provoking exertion, and again, if this was severe and sudden, the related facial and ocular phenomena would follow the onset of angina. The eyelid remained down for the whole day following an attack, but on waking the next morning the lid was normal or showed only slightly ptosis; the same result often followed a short sleep even in the afternoon after an attack. Ptosis occurred with all severe attacks and he began to use it as a signal that, unless he rested, severe pain would certainly follow.

He never had any double vision or impairment of vision during these attacks, until on one occasion the day after a severe attack he noticed persistent diplopia on looking upwards. Sweating of the left side of the face during attacks became increasingly prominent.

When he inhaled amyl nitrite the chest and arm pain gradually disappeared over a period of time up to about fifteen minutes, depending on their severity. The pain in the face disappeared simultaneously, but the drug had no effect on the weeping or the ptosis, the former lasting for about half to one hour after the pain cleared and the ptosis until next day, or at any rate until after a period of sleep. Careful enquiry showed that the weeping and ptosis was always accompanied by mild mid-sternal discomfort and dyspnoea, and always followed by facial pain and then by chest and arm pain unless he rested absolutely at the onset.

The patient was admitted under our care on July 16, 1945, and has remained under observation for three months. Physical examination on admission showed an obese subject with a pulse rate ranging between 60 and 80 a minute and a regular rhythm. The brachial vessels were thickened, but the heart size and sounds were normal and no murmurs were heard. The chest and abdomen showed no signs of disease and the nervous system was intact. No cranial bruits were heard. The blood pressure was 140/80, and the urine showed no abnormal constituents. Radiological examination showed no evidence of cardiac or aortic enlargement and there was no peripheral arterial calcification. The Wasserman reaction was negative. Cardiography showed left axis deviation and steep inversion of T III.

Three attacks were observed before relief was obtained with vasodilators: in symptomatology they were identical with those described by the patient. The following observations were made during the half-hour preceding the onset of chest pain. The pulse rate showed no change but the blood pressure rose to 170/110. There was flushing of the whole of the left face with hyperhydrosis, extreme conjunctival congestion, left pupillo-dilatation with retention of normal pupillary reactions (evident only in a dim light and therefore sympatheticotonic), striking dilatation of the retinal veins on ophthalmoscopy, and left ptosis of moderate degree and gradual onset. Electrocardiography during an attack showed inversion of T II in addition to the previous findings. The facial and ocular symptoms cleared within an hour of relief of the pain by amyl nitrite inhalation, except that the ptosis remained: next morning the ptosis was still evident though less. After the second and severest attack it persisted for four days and was associated with vertical diplopia. At this stage he was seen by Squadron-Leader R. S. Sampson, ophthalmologist, who reported left ptosis and weakness of the left superior rectus muscle as revealed by red-green glasses and the Maddox rod test. At the same time conjunctival instillation of one drop of $1 / 1000$ adrenalin, carried out at his suggestion, revealed left sympatheticotonia as shown by left pupillo-dilatation, the right pupil being unaffected. 
Radiological examination of the skull, including stereoscopic views of the pituitary region and the sphenoidal fissures showed no deviations from normal and in particular no abnormal calcification or vascular shadows.

At present (10/10/45), after prolonged rest in bed, he is free from symptoms except for occasional post-prandial and exertional dyspnoea, usually preceded by left lachrymation and conjunctival suffusion.

\section{Discussion}

The phenomena described above as preceding and accompanying the patient's attacks of angina pectoris are not easy to explain. The features shown in the face and eye appear to comprise two clearly defined components. First, there is evidence of irritation of the cervical sympathetic in lachrymation, hyperhydrosis, vasodilatation, pupillo-dilatation, and abnormal pupillary response to adrenalin. Secondly, there is evidence of a partial paralytic lesion of the third cranial nerve, in ptosis and paresis of the superior rectus muscle, producing diplopia.

Wide irradiation of different impulses in the spinal cord might be related to the first group of symptoms, but would not explain the second. Of intermittent pressure from a dilated or aneurysmal vessel within the skull the reverse is true. Yet the constant association and close time-relation of the two components of the syndrome indicate the probability of an equally close relation in their mode of production.

Consideration of the anatomical pathways involved suggests a possible resolution of this difficulty. The nervous pathways of cardiac pain are not completely understood, but are considered to pass mainly via the sympathetic afferents of the cardiac plexus to the middle and inferior cervical (stellate) ganglia and the cervical sympathetic chain. From here they pass into the cord by way of the white rami communicantes and the upper four thoracic and possibly the eighth cervical posterior roots, to terminate at the base of the posterior horn in relation to the origin of the spino-thalamic tract.

The sympathetic pupillo-dilator fibres originate in cells of the posterior hypothalamic nuclei, having connections there with cortical levels, and they pass down through the medulla and cervical spinal cord in close relation with the pyramidal tracts, to terminate in the ciliospinal centre, situated in the lateral horn of grey matter at the levels of the eighth cervical to the second thoracic segments. Here, preganglionic fibres originate, and, passing out with the lowest cervical and upper two thoracic anterior roots, make their way via the white rami communicantes to the inferior cervical (stellate) ganglia. These fibres course up the sympathetic chain to their cell-station in the superior cervical ganglia, where post-ganglionic fibres arise to be distributed to the eye by way of the coat of the internal carotid arteries and their branches. The sympathetic supply to the other parts concerned in Horner's syndromethe tarsal muscles, the muscle of Muller, and the facial sweat glands and blood vessels-follow a similar, if less clearly defined, route.

These two pathways, the one afferent and the other efferent, therefore have part of their course (between the stellate ganglion and the spinal cord) in common, except that the bulk of the afferent fibres probably pass in the posterior, and the bulk of the efferent fibres in the anterior roots of the same segments. More significantly, their cell-stations within the spinal cord are in close proximity. The cilio-spinal centre lies in the lateral horn of the grey matter between the eighth cervical and second thoracic segments, with the related sympathetic efferent cell-stations close by, while the synaptic connections between the cardiac visceral afferents and the cells of origin of the spino-thalamic tract lie at the base of the posterior horns at the same levels of the cord.

These anatomical considerations suggest that the sympathetic symptoms in the present case are produced in the spinal cord. MacKenzie's hypothesis of segmental and extrasegmental sensory irradiation is used to explain the wide somatic reference of anginal pain, which in this case involved all dermatomes from the third cervical to the first thoracic, and it is perhaps significant that the facial pain in the present case also indicates a very widespread irradiation. The peripheral site of this pain, affecting chiefly the temple, cheek, and jaw, and sparing the nose and the central parts of the face, suggests a central origin and is consonant with excitation of the lower part of the sensory nucleus of the fifth nerve which extends 
into the cord as far as the second cervical segment. It is known that referred cardiac pain may be accompanied by, and on occasion replaced by, sweating in the area of reference, while the salivation and polyuria that occasionally accompany angina pectoris can hardly be explained otherwise than as viscero-sensory reflexes similarly excited by irradiation from cardiac afferents.

In the present case, therefore, it is suggested that the sympatheticotonic phenomena were due to central irradiation within the spinal cord. The nature of the recurrent partial paresis of the oculo-motor nerve indicates a peripheral lesion, recoverable and probably involving only the superior ramus of the nerve that supplies the two muscles involved (levator palpebrae superioris and superior rectus). The delayed onset and slow recovery of the third nerve signs supports a mechanical causation, and we suggest that the paresis may have been due to local pressure on the superior ramus of the nerve by a dilated artery. The anterior site of the third nerve involvement, and the failure to elicit any evidence of impaired function in the first division of the fifth nerve on repeated testing during and between attacks, suggests that the ophthalmic artery or one of its branches rather than the internal carotid was responsible, and in its distension both the widespread sympathetic vasodilatation and the preanginal rise in blood pressure may have played a part.

\section{CoNCLUSIONS}

A case of angina pectoris with two unusual groups of associated symptoms is described. In the first there was evidence of irritation of the cervical sympathetic, as shown by lachrymation, hyperhydrosis, vasodilatation, pupillo-dilatation, and an abnormal pupillary response to adrenalin. In the second there was evidence of a partial paralytic lesion of the third cranial nerve resulting in ptosis and paresis of the superior rectus muscle, producing diplopia.

The possible mechanisms have been discussed. It is suggested that the sympatheticotonic phenomena are due to central irradiation within the spinal cord and that the recurrent partial paresis of the oculo-motor nerve may be due to local pressure on the superior ramus by a dilated opthalmic artery.

We wish to thank Squadron Leader R. S. Sampson for many helpful suggestions in relation to this case. 